\title{
Vorwort zur neunzehnten Auflage
}

Als Anhang 3 sind das Gesetz über Straßenwesen von 1934 und die Durchführungsverordnung dazu wieder aufgenommen worden, nachdem ihr Fortbestehen trotz $\S 25$ FStrG nunmehr geklärt worden ist. - Anhang 6 bringt die Verordnung von 1955 über die Uberwachung der Selbstfahrermietfahrzeuge. - Das (internationale) Protokoll über Verkehrszeichen (1949) ist hinter dem (zweiten) Anhang 7 wiedergegeben, der das schon in die 18. Auflage aufgenommen gewesene internationale Verkehrsabkommen (1949) enthält; dieses neue Abkommen ist jetzt mit Erläuterungen versehen worden. Ferner enthalten die deutschen Vorschriften der Zulassungs- und der Verkehrs-Ordnung jeweils Hinweise auf die entsprechenden internationalen und umgekehrt.

Von der durchgesehenen recht umfangreichen Rechtsprechung sind an Obergerichtsurteilen mehr als 490 verwertet worden. Die neuere Rechtsprechung gestattete vielfach Ausmerzung solcher älterer Entscheidungen, die nicht zur Förderung des Verständnisses des Gewordenen beizubehalten waren.

Zum Recht der Fahrräder mit Hilfsmotor veranlaßte die Klarstellung in der V v 17.11. 54 erneute Umarbeitung insb der Erläuterungen zum § 27 StVG. Auch sonst haben Rechtsprechung und Gesetzgebung Umarbeitungen verursacht, deren Umfang daraus erhellt, $\mathrm{da} B$ aus der 18. Auflage von den Seiten 415-662 der Zulassungs-Ordnung nur rund 1/s sachlich unverändert geblieben ist, von den Seiten 663-916 der Verkehrs-Ordnung gar nur rund $1 / \%$.

Bei dem durch Rechtsprechung und Gesetzgebung verursachten Wachsen des Umfangs des Stoffes ist Maßhalten in der Einbeziehung der Rechtsgebiete zwingende Notwendigkeit; s Vorwort zur 17. Auflage; mehr als Herstellung des Zusammenhangs zu den nicht unmittelbar zum Straßenverkehr gehörenden Rechtsgebieten verbietet der Raummangel; trotzdem vermißt mancher Kritiker entweder die Darstellung solcher Rechtsgebiete oder ihre Vertiefung. Umgekehrt beanstandet manche Kritik ein Zuviel, zB, als nach Meinung des Rezensenten nicht zum Straßenverkehr gehörig, die Berücksichtigung des PflVersG, ja sogar die des nunmehrigen $\S 248 \mathrm{~b}$ StGB, also des Nachfolgers der seit der 9. Auflage behandelten (früheren) $\mathrm{V}$ gegen den unbefugten Gebrauch von Kfzn und Fahrrädern $\checkmark$ 20.10.32. Den goldenen Mittelweg zwischen dem Zuwenig und dem Zuviel zu halten, ist nicht leicht; und jedem Kritiker „Recht getan ist eine Kunst, die keiner kann”.

Gegenüber vereinzelter Kritik erwähnte das Vorwort zur 17. Auflage, die Herstellungsdauer eines umfangreichen Buches schließe Berücksichtigung aller Neuerungen bis zum Erscheinen einer neuen Auflage selbst dann aus, wenn auch noch während der Herstellung nach Möglichkeit Neues berücksichtigt werde. Trotz dieses Hinweises wurde an der 17. Auflage und trotz seiner Wiederholung im Vorwort der 18. wurde auch an dieser 
Auflage wiederum gleichartige Kritik geübt. Der Verfasser wird nunmehr darauf verzichten, auf solche Kritik einzugehen.

Wenn (kein Einzelfall!) Kritiken zur 18. Auflage Berücksichtigung, von Urteilen vermissen, so wird dem Verfasser kaum ein berechtigter Vorwurf daraus zu machen sein, daß er die vermißten Entscheidungen an den von ihm für richtig gehaltenen Stellen gebracht hat und nicht da, wo, für ihn nicht voraussehbar, die Kritik sie (vergeblich) sucht.

Die laufend ausgewerteten Zeitschriften sind bis zu den jeweils dabei vermerkten Heften berücksichtigt worden: Deutsches Autorecht, Recht des Kraftfahrers und Verkehrsrechtliche Mitteilungen Heft 5; Verkehrsrechtssammlung Bd 8 Heft 5; Neue Juristische Wochenschrift Heft 23; Verkehrsblatt Heft 10; Zeitschrift f Versicherungswesen Heft 11. 